\title{
SPECIAL ARTICLE COVID-19: trainee perspectives from unprecedented changes on the Paediatric Intensive Care Unit (PICU)
}

\author{
Naren Siva ${ }^{1}$, Philip Knight ${ }^{1}$ and Akash Deep ${ }^{1,2}$
}

During the coronavirus disease 2019 (COVID-19) global pandemic, there has been a need to develop surge capacity. Since the disease is uncommon in children, working on a paediatric intensive care unit (PICU) has required an expansion of roles and responsibilities outside established confines. The most drastic change in practice involved having to care for both critically ill adults and children side by side on the PICU. Redeployment to work on an adult critical care unit as required was similarly momentous. Based on our experience of managing this surge in one of the UK's worst hit tertiary hospitals, we are sharing our reproducible approaches that benefitted trainees. This will be relevant to paediatricians globally who are assisting in critical care strategies and future pandemic planning.

Pediatric Research (2022) 91:70-71; https://doi.org/10.1038/s41390-021-01418-5

The coronavirus disease 2019 (COVID-19) global pandemic has presented all of us with various unprecedented challenges. Trainee doctors, who form an important and substantial part of the medical workforce, have faced many novel predicaments. In our workplace, these varied vastly from dealing with sudden rota changes, redeployment, working on a hybrid intensive care unit (ICU) for adults and children and adjusting to new teaching methods.

In March, as a cohort of trainees we began our paediatric intensive care unit (PICU) rotation, a 6-month placement within our 8-year specialty training programme. For the majority, it was our first direct experience in this subspecialty and so naturally, alongside excitement, there was a level of disquietude. It rapidly became evident, though, with numerous incoming COVID-19related emails (including notification of the paediatric intensive care society and intensive care society joint statement ${ }^{1}$ ) that this placement was not going to be as originally anticipated. Among these emails, the early guidance on the proper use and testing of personal protective equipment was a boost. This aided in resisting trepidations of becoming infected at work $^{2}$ and potentially passing the virus onto others.

A common grave apprehension that occupied us was not just the thought of looking after seriously ill patients in new conditions but additionally managing an unfamiliar patient group. Anticipatory adult advanced life support resuscitation training received was therefore invaluable. Having this refresher and then building on this in simulation case scenarios was greatly valued. The essential relevant knowledge and confidence gained empowered us in future unfamiliar critical care situations.

It was soon communicated that the PICU would be amalgamated to look after adult patients and that trainees would occasionally be redeployed to an adult critical care unit. In advance of this uncomfortable encroaching reality, lectures and virtual meetings were delivered giving an introduction to adult critical care. With a broad array of topics to digest and absorb, there was appreciation for tips received to help us prepareparticularly useful were memorable mnemonics linked to daily checklists and assessments. Assimilating this crucial information was assisted by the availability of online learning modules. The continual accessibility of up-to-date clinical guidelines through a smartphone app, Clinibee, was a vital resource. Having direct access at hand to this both at the bedside and outside of the hospital to keep up with current and emerging practices was allimportant.

Despite the tremendous benefits attributable to the wideranging training and learning provided, nerves still persisted around working in a new capacity outside of usual remits. It was therefore essential after first stepping onto the adult critical care unit to invest time in becoming familiar with the unaccustomed surroundings. The perpetual accessible support on the adult ICU was a reassuring benefit. This allowed the focus to be on delivering safe patient care. It also relieved the pressure from connotations of needing to act outside of our clinical competences.

The uniqueness of the PICU becoming a hybrid unit presented another prospectively daunting challenge. This was a distinct model as a number of other PICUs had completely converted themselves into adult ICUs. Managing critically ill children and adults on the same floor by the same set of doctors and nurses was novel. It was therefore paramount to always maintain awareness and adaptability when imparting care in view of the greatly differing physiologies among the patients. The resolve and capacity to face these struggles were augmented through an approachable and supportive network of multidisciplinary healthcare professionals. This significantly aided with providing optimum care for all patients on the PICU.

A new COVID-19 rota added a sense of comradery. Those on the reserve rota readily volunteered to cover for enforced absences,

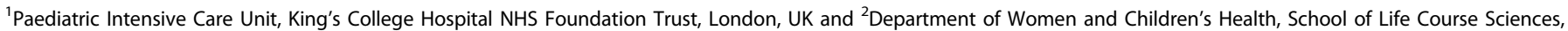
King's College London, London, UK

Correspondence: Naren Siva (naren.siva@nhs.net)
}

Received: 28 July 2020 Revised: 22 January 2021 Accepted: 1 February 2021

Published online: 2 March 2021 
which gave an assurance of safe staffing levels. The dismay at having reduced clinical exposure, due to the change in the rota, meant it was important to sustain a proactive attitude. Utilisation of this unexpected non-clinical time with pertinent support enabled trainees to become involved in research projects. With the strains of high levels of exhaustive workloads and social distancing guidance, there were concerns about the opportunities for continued education. Adapting to schedule regular virtual teaching was merited by both trainees and clinical supervisors.

\section{CAN THIS EXPERIENCE BE EXTRAPOLATED TO OTHER TRAINEES TO COPE WITH CHANGES IN WORKING ENVIRONMENTS AND PATIENT POPULATIONS IN AN UNPRECEDENTED PANDEMIC SITUATION?}

The adoption of a hybrid model and redeployment posed notable shared unease among trainees. ${ }^{3}$ This included our clinical knowledge gap and at the extreme the thought of negligence. Other causes of consternation in the team were inexperience with specific equipment and pharmacy protocols. Furthermore, there were collective anxieties related to caring for the two distinct patient groups on the same unit. A significant overall concern related to emotional resilience. When considering these, it was important to remember, though, that the support we provided our fellow healthcare professionals in those specialties under the greatest stress from the pandemic was critical.

In our experience, the hybrid model alleviated certain safety risk factors as it enabled existing PICU staff to care for adult patients within familiar settings and with known personnel. It also minimised the need for redeployment. A beneficial factor that helped in the success of this model was collaboration. This included instigating the routine attendance of senior adult ICU staff at handovers and huddles on the PICU. Additionally, adult ICU staff always remained readily accessible to assist the PICU team.

On reflection, there are key lessons that would be applicable to fellow trainees when faced with similar circumstances. Pivotal steps in advance of any transition and transformations are as early as possible identifying and reviewing the responsibilities and demands of a revised role. With this understanding, generic transferable clinician skills can then be built on. This should be through acquainting with relevant guidelines and practices, gaining adequate information on new working environments and pursuing opportunities to participate in any necessary additional training. ${ }^{4}$ Upskilling after an initial induction should be ongoing and guidelines must also be made easy to access. When at the boundaries of a comfort zone and in the context of heightened workloads, it is salient to be constantly conscious of our limitations. Patient care decisions should always be made in their best interests, ${ }^{5}$ and so there should be continued consideration of both competence and fatigue. Building a rapport with new colleagues should also be an initial focus. This includes informing them of your personal clinical capabilities and competencies. This will mean that they are aware of the assistance different individuals may need and how someone's existing knowledge and skills could be best utilised in the team. Clinical supervision is important. Trainees must be able to recognise who is responsible for their supervision and be content with the supervision arrangements in place. Knowing in a new placement how and who to approach for advice and supervision is essential. Trainees should not be apprehensive in asking supervisors for instruction, feedback or support, including when dealing with fears and anxieties. Anticipating the toll from working in extremely unwonted conditions, it is fundamental that we do not overlook caring for the welfare of colleagues and our own health (physical and mental). ${ }^{6}$ Within our hospital, the provision of staff wellbeing hubs was a positively received intervention.

The unpredicted and unfortunate circumstances associated with the first wave of COVID-19 had the potential to lead to a disconcerting experience for trainees on the PICU. In this demanding period, there were no universal pre-existing plans. It was still possible, though, for us as trainee paediatricians to develop a level of assuredness, competency and flexibility to meet population needs. We were thus able to work in reorganised care environments that encompassed patient cohorts that are exceptionally atypical from our routine practice. Highly influential was the efficient and efficacious preparation received through dedicated induction and training combined with sustained comprehensive levels of support on all matters. This wholly strengthened our resilience to conduct committed professional duties and enhance our career long learning.

Now in the midst of a second wave that has caused more hospitalisation and ICU admissions, we have been able to use these learned lessons to be better prepared. This time there was earlier education and training and also the involvement of a wider pool of paediatric trainees, including those who regularly work outside of the PICU. This has meant that there has been better organisation in the assigning of non-ICU support roles and the ability to maintain continuity of care when facing even higher levels of sickness.

\section{ACKNOWLEDGEMENTS}

The authors would like to thank all the staff of both the PICU and adult ICU departments of King's College Hospital NHS Foundation Trust. No funding source was secured for this article.

\section{AUTHOR CONTRIBUTIONS}

N.S. and A.D. conceptualised the idea for the topic. N.S. wrote the initial manuscript. N.S., A.D. and P.K. contributed to revisions. All authors approved the final version of the manuscript.

\section{ADDITIONAL INFORMATION}

Competing interests: The authors declare no competing interests.

Patient consent: Not required.

Publisher's note Springer Nature remains neutral with regard to jurisdictional claims in published maps and institutional affiliations.

\section{REFERENCES}

1. PICS. PICS and ICS Joint Position Statement, 2020. https://picsociety.uk/news/picsand-ics-joint-positionstatement-12-mar-2020/ (2020).

2. Wang, J., Zhou, M. \& Liu, F. Reasons for healthcare workers becoming infected with novel coronavirus disease 2019 (COVID-19) in China. J. Hosp. Infect. 105, 100-101 (2020).

3. Academy of Medical Royal Colleges. Plans regarding trainee redeployment during the COVID-19 pandemic: a position statement from the Academy trainee doctors group, 2020. https://www.aomrc.org.uk/wp-content/uploads/2020/03/200326_ATGD_COVID19-redeployment.pdf (2020).

4. Faculty of Intensive Care Medicine. \#BetterTogether: the critical care team. https:// www.ficm.ac.uk/sites/default/files/better_together_-_final_0.pdf (2020).

5. General Medical Council. Good Medical Practice (General Medical Council, 2019).

6. Greenberg, N., Docherty, M., Gnanapragasam, S. \& Wessely, S. Managing mental health challenges faced by healthcare workers during covid-19 pandemic. BMJ 368, m1211 (2020). 